\title{
Modeling software architecture design on data storage security in cloud computing environments
}

\author{
Jagdish Mukta, Viloria Amelec, Vargas Jesus, Pineda Lezama Omar Bonerge, Ovallos- \\ Gazabon David.
}

\begin{abstract}
Cloud-based computation is known as the source architecture of the upcoming generation of IT enterprise. In context to up-coming trade solutions, the Information Technology sections are established under logical, personnel, and physical control, it transfers application software and large database to appropriate data centers, where security and management of database with services are not trustworthy fully. So this process may face many challenges towards society and organizations and that not been well understood over a while duration. This becomes one of the major challenges days today. So in this research, it focuses on security-based data storage using cloud, which plays one of the important aspects bases on qualities of services. To assure user data correctness in the cloud system, a flexible and effective distributed technique with two different salient features was examined by utilizing the token called homomorphic with erasure-coded data for distributed verification, based on this technique it achieved error data localization and integration of storage correctness. Also, it identifies server misbehaving, efficient, and security-based dynamic operations on data blocking such as data append, delete, and update methods. Performance analysis and security show the proposed method is more effective resilient and efficient against Byzantine failure, even server colluding attacks and malicious data modification attacks.
\end{abstract}

\section{Palabras clave}

Cloud security, Architecture design, Data storage, Homomorphic token, Dynamic operation. 